Ambient temperature regulates the expression of a small set of sRNAs influencing plant development through $N F-Y A 2$ and $Y U C 2$

Péter Gyula ${ }^{1, \dagger} *$, Ivett Baksa ${ }^{1, \dagger}$, Tamás Tóth ${ }^{1}$, Irina Mohorianu ${ }^{2,3}$, Tamás Dalmay ${ }^{2}$, György

\title{
Szittya $^{1} *$
}<smiles>C1CC1</smiles><smiles>[TlH]</smiles>

${ }^{1}$ National Agricultural Research and Innovation Center, Agricultural Biotechnology Institute,

Epigenetics Group, Szent-Györgyi A. 4., Gödöllö H-2100, Hungary

${ }^{2}$ School of Biological Sciences, University of East Anglia, Norwich, United Kingdom

${ }^{3}$ School of Computing Sciences, University of East Anglia, Norwich, United Kingdom

${ }^{\dagger}$ These authors contributed equally to this work

\section{E-mails:}

Péter Gyula - gyula.peter@abc.naik.hu

Ivett Baksa - baksa.ivett@abc.naik.hu

Tamás Tóth - toth.tamas@abc.naik.hu

Irina Mohorianu - I.Mohorianu@uea.ac.uk

Tamás Dalmay - T.Dalmay@uea.ac.uk

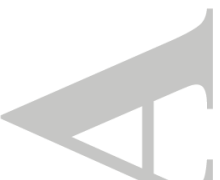

This article has been accepted for publication and undergone full peer review but has not been through the copyediting, typesetting, pagination and proofreading process which may lead to differences between this version and the Version of Record. Please cite this article as doi: 10.1111/pce. 13355 


\section{Corresponding Authors:}

\section{Dr. György Szittya}

Institute of Plant Biotechnology,

Agricultural Biotechnology Institute,

National Agricultural Research and Innovation Centre

Szent-Györgyi Albert út 4.

\section{H-2100, Gödöllő}

Hungary

Phone: +36-28-526230

Fax: +36-28-526101

E-mail: szittya.gyorgy@abc.naik.hu

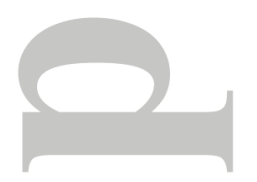

\section{Dr. Péter Gyula}

Institute of Plant Biotechnology,

Agricultural Biotechnology Institute,

National Agricultural Research and Innovation Centre

Szent-Györgyi Albert út 4. 
H-2100, Gödöllö

Hungary

Phone: $+36-28-526230$

Fax: +36-28-526101

E-mail: gyula.peter@abc.naik.hu

Ambient temperature regulates the expression of a small set of sRNAs influencing plant development through $N F-Y A 2$ and $Y U C 2$

Running title: Study of Arabidopsis thermoresponsive sRNA classes

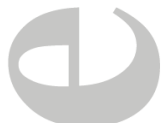

\section{Highlight}

Comparison of sRNA populations at 15,21 and $27{ }^{\circ} \mathrm{C}$ in Arabidopsis thaliana revealed that miR169, targeting NF-YA2 and a hc-siRNA locus regulating YUC2 might have a role in temperature adaptation. 


\section{Abstract}

Plants substantially alter their developmental program upon changes in the ambient temperature. The 21-24 nt small RNAs (sRNAs) are important gene expression regulators, which play a major role in development and adaptation. However, little is known about how the different sRNA classes respond to changes in the ambient temperature. We profiled the sRNA populations in four different tissues of Arabidopsis thaliana plants grown at 15,21 and $27{ }^{\circ} \mathrm{C}$. We found that only a small fraction $(0.6 \%)$ of the sRNA loci are ambient temperaturecontrolled. We identified thermoresponsive miRNAs and identified their target genes using degradome libraries. We verified that the target of the thermoregulated miR169, NF-YA2, is also ambient temperature-regulated. NF-YA2, as the component of the conserved transcriptional regulator NF-Y complex, binds the promoter of the flowering time regulator $F T$ and the auxin biosynthesis gene $Y U C 2$. Other differentially expressed loci include thermoresponsive phased siRNA loci that target various auxin pathway genes and tRNA fragments. Furthermore, a temperature dependent 24-nt heterochromatic siRNA locus in the promoter of $Y U C 2$ may contribute to the epigenetic regulation of auxin homeostasis. This holistic approach facilitated a better understanding of the role of different sRNA classes in ambient temperature adaptation of plants.

Keywords: ambient temperature, Arabidopsis thaliana, degradome, miRNA, phasiRNA, siRNA, tRNA fragments, NF-YA2, YUC2. 


\section{Brief Summary}

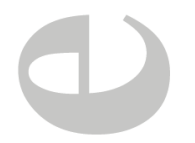

Our previous study on temperature-dependent antiviral silencing suggested that the endogenous silencing regulating plant development is also under temperature control. Surprisingly, the comparison of sRNA populations at 15,21 and $27{ }^{\circ} \mathrm{C}$ in Arabidopsis thaliana seedlings, roots, leaves, and flowers revealed that only a small fraction of the endogenous small RNAs is temperature-regulated. We found that miR169, targeting NF-YA2 and JAZ4, and a hc-siRNA locus regulating YUC2 might have a role in temperature adaptation.

\section{Introduction}

Since plants are sessile organisms, it is vital for their survival to readily adapt their growth and development in response to a continuously changing environment. Temperature is a key environmental signal, and accurate monitoring of ambient temperature is fundamental for living organisms. Plants measure temperature very accurately and can show remarkable responses to small changes in temperature (Argyris et al. 2005). Temperature can dramatically affect plant growth and development; for example, in response to higher temperatures plants grow faster and flower earlier, while plants grown in the cold remain smaller and more compact.

Various mechanisms have been proposed for ambient temperature sensing in plants. Recently, the dark-reversion of the red/far-red photoreceptor PHYTOCHROME B (PHYB) has been shown to be temperature dependent (Jung et al. 2016; Legris et al. 2016), which affects the interaction with the PHYTOCHROME-INTERACTING FACTOR 4 (PIF4), a 
critical regulator of thermomorphogenesis (Quint et al. 2016) and possibly the circadian Evening Complex that is a transcriptional co-ordinator of endogenous and environmental signals in Arabidopsis thaliana (Ezer et al. 2017a). Epigenetic factors have also been implicated in conveying thermosensory information. In a forward genetic screen in Arabidopsis thaliana, chromatin has been found to play a major role in the detection of changes in ambient temperature (Kumar \& Wigge 2010). According to this study, nucleosomes containing the alternative histone H2A.Z provides thermosensory information for the transcriptome. Nucleosomes play an active role in controlling gene expression through modulating the ability of transcription factors to access their cis elements (Lam, Steger \& O'Shea 2008; Segal \& Widom 2009). H2A.Z-containing nucleosomes exhibit a much tighter wrapping of their DNA than canonical H2A nucleosomes. The occupancy of H2A.Zcontaining nucleosomes decreases with temperature, which affects gene expression. The same effect was observed in budding yeast, indicating that this is an evolutionarily conserved mechanism in eukaryotes (Kumar \& Wigge 2010).

Small RNAs (sRNAs) have been recognized as important gene expression regulators which can act both transcriptionally and post-transcriptionally (Carthew \& Sontheimer 2009; Voinnet 2009). The function of sRNAs is to give sequence specificity to effector complexes. This layer of gene regulation is known as RNA silencing. Most of the plant sRNAs are 2124-nt long, and they belong to two major classes: microRNAs (miRNAs) and short interfering RNAs (siRNAs). All sRNA are generated by an RNase-III type enzyme called DICER-LIKE ( $D C L$ ) from double-stranded RNA (dsRNA), but dsRNA can be formed through different mechanisms. MiRNAs are produced from precursor RNA (pre-miRNA) where single-stranded primary transcripts form a hairpin-like structure which is subsequently cleaved during a stepwise process in the cell nucleus by DCL1. The released miRNA duplex 
is transported to the cytoplasm, where one of the miRNA strands is incorporated into ARGONAUTE 1 (AGO1) and the other strand is eliminated (Rogers \& Chen 2013). Plant miRNAs have near-perfect complementarity to their targets, and they bind to the target sites to guide the cleavage (Llave, Xie, Kasschau \& Carrington 2002), although there are examples where the translation of the mRNA is suppressed without a cleavage (Aukerman \& Sakai 2003; Chen 2004). MiRNAs are mainly 21-nt-long and are important regulators of endogenous gene expression during development and environmental adaptation as it has been demonstrated by the pleiotropic developmental abnormalities seen in many miRNA biogenesis mutants (Voinnet 2009).

The other class of sRNAs, based on their biogenesis, is the short interfering RNAs (siRNAs). SiRNAs are produced from a long dsRNA precursor. They can be categorized into several different groups. Based on the origin of the dsRNA and their targets the two most important ones are the following:

(1) Phased siRNAs (phasiRNAs) are 21-nt-long and require components of both the miRNA and siRNA pathways for their biogenesis. They are produced from transcripts, which are converted into dsRNA by RNA-dependent RNA Polymerase 6 (RDR6) after a miRNAguided cleavage (Peragine, Yoshikawa, Wu, Albrecht \& Poethig 2004; Vazquez et al. 2004), usually by a 22-nt miRNA-guided AGO (Fei, Xia \& Meyers 2013). The dsRNA is then diced in a phased manner by DCL4 to generate 21-nt phasiRNAs (Gasciolli, Mallory, Bartel \& Vaucheret 2005; Xie, Allen, Wilken \& Carrington 2005; Yoshikawa, Peragine, Park \& Poethig 2005). The phase of the dicing reaction is determined by the initial miRNA cleavage site. PhasiRNAs can be generated from either coding or non-coding transcripts. The first phasiRNAs were discovered in Arabidopsis thaliana, and they were all produced from noncoding transcripts called TAS RNAs (trans-acting siRNAs), and therefore phasiRNAs were 
initially called ta-siRNAs. Later it was discovered that in addition to the non-coding TAS genes, protein-coding genes such as NBS-LRR and pentatricopeptide repeat (PPR) genes are also targeted by 22-nt long miRNAs and produce phased siRNAs. Therefore, a more general name was coined: phasiRNAs (Zhai et al. 2011). PhasiRNAs can similarly target mRNAs as miRNAs.

(2) The vast majority of endogenous siRNAs form a complex population of more than 100,000 different siRNAs transcribed from thousands of loci (Kasschau et al. 2007; Mosher, Schwach, Studholme \& Baulcombe 2008). A subset of these 24-nt cis-acting siRNA molecules are involved in stress responses, others target DNA and cause changes in chromatin structures. The biogenesis of these heterochromatic siRNAs requires an RNA polymerase IV (Pol IV), which is unique to the plant kingdom and homologous to the DNAdependent RNA polymerase II (Zhang, Henderson, Lu, Green \& Jacobsen 2007). Pol IV transcribes single-strand precursor RNAs from non-random sites throughout the genome, mostly at repetitive or transposable elements, or in the vicinity of transcription start sites and other cis-regulatory elements. The RNA precursors are immediately converted into doublestranded RNAs by RNA Dependent RNA Polymerase 2 (RDR2). These dsRNAs are then cleaved into 24-nt siRNAs by DCL3, and the resulting siRNAs are loaded into a different set of ARGONAUTE proteins than the miRNAs (AGO 3, 4, 6, 9) in the cytoplasm, and then transported back into the nucleus, to target cytosine methylation at cognate genomic DNA sequences. This downstream methylation-targeting step of the pathway requires a second RNA polymerase, Pol V. It is another plant-specific RNA polymerase with an AGO-binding motif. It generates nascent scaffold transcripts that serve as platforms for complexes of RNA directed DNA methylation (RdDM) in Arabidopsis, likely through base-pairing between 
AGO4/siRNA complexes and scaffold RNA (Wierzbicki, Ream, Haag \& Pikaard 2009;

Zhong et al. 2014; Matzke, Kanno \& Matzke 2015; Zhou \& Law 2015).

Small RNAs are not only important in endogenous gene regulation, but they also play a role in plant defense against molecular parasites, including viruses (Ding \& Voinnet 2007).

Environmental factors, especially temperature, have a strong influence on the outcome of plant-virus interactions. We described previously that siRNA-mediated plant defense against molecular parasites is temperature-dependent (Szittya et al. 2003). At low ambient temperature $\left(15^{\circ} \mathrm{C}\right)$ the level of virus or transgene-derived siRNAs is dramatically reduced, and as a consequence, this led to enhanced virus susceptibility or a loss of gene silencingmediated transgenic phenotypes. In contrast, with a temperature rising, RNA silencing is activated, and the amount of siRNAs gradually increases. Furthermore, in a miRNA expression study, researchers analyzed the expression levels of 120 unique Arabidopsis thaliana miRNAs in response to ambient temperature changes $\left(16\right.$ and $\left.23^{\circ} \mathrm{C}\right)$ by using miRNA microarray and miRNA northern hybridization analysis (Lee et al. 2010). They identified six ambient temperature-responsive miRNAs (miR156, miR163, miR169, miR172, miR398, and miR399).

Since we and others showed that low temperature inhibits sRNA-mediated defense and some of the endogenous sRNAs show temperature-dependent expression, we assumed that they likely have a role in temperature-dependent signaling. We tested this hypothesis by highthroughput sequencing of sRNAs from seedling, root, leaf, and flower of Arabidopsis thaliana plants grown at 15,21 and $27^{\circ} \mathrm{C}$. We identified 50 temperature-regulated miRNA sequences (including $5 p$ and $3 p$ sequence variants) and 48 miRNA/transcript pairs using degradome libraries. Among the temperature-regulated miRNA targets, we identified the $N F$ YA2, a component of the NF-Y transcriptional complex that regulates the flowering time 
integrator FT and many other genes. We also found some thermoregulated phasiRNAs that target elements of the auxin signaling pathway. Furthermore, we identified a temperaturedependent 24-nt heterochromatic siRNA locus that is likely to contribute to the epigenetic regulation of the auxin biosynthesis gene $Y U C 2$.

\section{Materials and methods}

\section{Plant materials, growth conditions, and sample collection}

Arabidopsis thaliana Col-0 ecotype plants were grown at 15,21 or $27^{\circ} \mathrm{C}$ in plant growth chamber (SANYO/Panasonic MLR-352-PE) under standard long-day conditions (16L:8D). Plants were either grown on half-strength Murashige and Skoog plates including vitamins under sterile conditions (for seedling and root samples) or in soil (for the leaf and flower samples). Both plates and pots were kept at $4{ }^{\circ} \mathrm{C}$ for three days to synchronize germination. The seedling samples were collected at stage 1.04 (Boyes et al. 2001), which was day 9 at 27 ${ }^{\circ} \mathrm{C}$, day 11 at $21{ }^{\circ} \mathrm{C}$, and day 15 at $15^{\circ} \mathrm{C}$ (Fig S1, panel A). The seedlings for the root samples were grown on vertical plates to ease the collection of the roots (Fig S1, panel B). The root samples were collected at day 11 at $27{ }^{\circ} \mathrm{C}$, day 14 at $21{ }^{\circ} \mathrm{C}$ and day 20 at $15^{\circ} \mathrm{C}$. For the leaf samples, mature rosette leaves of plants before flowering (stage 3.50-3.70) were harvested (Fig S1, panel C). The leaf samples were collected at day 19 at $27^{\circ} \mathrm{C}$, day 23 at 21 ${ }^{\circ} \mathrm{C}$ and day 53 at $15^{\circ} \mathrm{C}$. For the flower samples, whole inflorescences of flowering plants (stage 6.10-6.30) were used (Fig S1, panel D). All samples were collected at around ZT6 (zeitgeber time, 6 hours after lights on) since the time of the day has a major impact on temperature-dependent transcription (Ezer et al. 2017a). RNA isolation was carried out from fresh tissues based on the Kaper and White phenol/chloroform extraction method (White \& 
Kaper 1989). The genomic DNA was isolated using the DNeasy Plant Kit (Qiagen) following the manufacturer's instructions. The quality and the quantity of the isolated nucleic acids were checked using a Nanodrop spectrophotometer and the integrity of DNAs or RNAs was checked via non-denaturing agarose gel electrophoresis.

\section{Preparation of the sequencing libraries}

Sequencing libraries for the sRNA, degradome, and RNA-seq were prepared from four tissues (seedling, root, leaf, flower) at three temperatures $\left(15,21,27^{\circ} \mathrm{C}\right)$ in two biological replicas in the case of sRNA and degradome libraries and without replicas in the case of the RNA-seq libraries. For the sRNA libraries, 1-5 $\mu \mathrm{g}$ total RNA was separated on a denaturating PAGE gel, and the sRNA fraction (approximately 10-40-nt RNAs) was isolated and purified from the gel. This enriched sRNA pool was used as a template for the next step. The sRNA libraries were constructed using the TruSeq Small RNA Sample Prep Kit (Illumina, CA, US), according to the manufacturer's instructions. The degradome libraries were prepared following the procedures previously described by Baksa et al. (Baksa \& Szittya 2017). The mRNA libraries for RNA-Seq were generated from $1 \mu \mathrm{g}$ total RNA using TruSeq RNA Sample Preparation Kit (Illumina, San Diego, CA, USA) according to the manufacturer's protocol (with polyA-selection). All types of libraries were sequenced on HiScan SQ platform (50 bp, single-end) but for the degradome libraries, a custom-made sequencing primer was utilized (Pantaleo et al. 2010).

Preprocessing of the raw data

Raw sRNA, degradome sequences in fastq format were processed and filtered with cutadapt 1.16 (Martin 2011) set to trim the Illumina sRNA adapter, keeping sequences with lengths between 18-35 nt that contain residues with quality higher than 20 . Untrimmed sequences 
(containing no or incomplete adaptor) were discarded. In the case of the RNA-seq libraries, no adaptor trimming was necessary because we got them already trimmed. The quality check was performed using FastQC 0.11.3 (Andrews 2010). The mapping statistics for all the libraries can be found in Table S1.

\section{sRNA locus prediction}

De novo sRNA locus prediction was carried out using ShortStack 3.4 (Johnson, Yeoh, Coruh \& Axtell 2016), using the Arabidopsis thaliana TAIR10 reference genome allowing one mismatch (full alignment without gaps), not considering reads that mapped to more than 1000 places in the genome. Manipulation of the alignment files was carried out with samtools 1.3.1 (Li et al. 2009). Summary statistics were prepared by a custom Linux shell script. Loci were annotated with bedtools 2.17.0 (Quinlan \& Hall 2010), using TAIR10 annotations as a reference. Filtering of the loci by ShortStack-assigned tags was carried out using a custom R script. During this filtering, heterochromatic siRNA loci were defined as having a Dicercall parameter of 24 (dominant size class), and not having an overlap with miRNA genes. PhasiRNA-producing loci were accepted when the Dicercall and PhaseSize parameters were 21, the PhaseScore was at least 25 (see ShortStack manual for the calculation of PhaseScore), and not having an overlap with miRNA genes. The phasiRNA sequences and the phaseinitializing miRNAs were determined with PhaseTank (Guo, Qu \& Jin 2015). The differential expression analysis of the sRNA loci between temperatures was carried out using DESeq2 (Love, Huber \& Anders 2014), separately for every tissue. We considered a locus expression changed when the normalized abundance $>=10$ across all samples in a tissue, the absolute fold-change was at least 1.5 and the adjusted p-value was lower than 0.05 . The p-value adjustment was performed by DESeq2 according to the Benjamini-Hochberg method 
(Benjamini \& Hochberg 1995). Plots and heat maps were generated using various $\mathrm{R}$ packages.

Micro RNA profiling and degradome analysis

MiRNAs were identified by merging known Arabidopsis thaliana miRNA sequences from miRBase v21 (Kozomara \& Griffiths-Jones 2014) and ShortStack-predicted mature and star sequences. The abundances of the miRNAs were determined across libraries. To make the read counts in different libraries comparable, we applied a normalization method that was suggested by McCormick et al. for sRNA sequencing projects (McCormick, Willmann \& Meyers 2011). Highly abundant and variable sources like reads aligned to structural RNAs (rRNA, tRNA, snRNA, snoRNA) or organellar (mitochondrial and chloroplastic) genomes were excluded from the calculation of the normalization factor but were not discarded. Using these effective library sizes read counts were scaled to one million reads (RPM).

The secondary structures of the newly identified candidate miRNAs were predicted and visualized with the RNA/Folding Annotation tool of the UEA Small RNA Workbench (Stocks et al. 2012). The temperature-regulated sRNAs were identified by filtering for sequences with a maximum normalized abundance of at least 40 RPM and with a minimum fold-change of 1.5 between at least one of two successive temperatures in at least one tissue. Degradome analysis was performed with CleaveLand4 (Brousse et al. 2014). Only hits of category 0-2 and with an Allen score 0-5 (Allen, Xie, Gustafson \& Carrington 2005) were retained. GO-term enrichment analysis of the target gene lists was performed with AgriGO server (Du, Zhou, Ling, Zhang \& Su 2010). First, we used all the TAIR9 annotated genes as the background. The Chi-square test was applied to determine the p-value. The p-values were corrected for multiple testing according to the Benjamini-Hochberg method. Next, all the miRNA target genes were used as a background. In this case, the Fisher exact test was 
applied to determine the p-value, as recommended by the AgriGO server documentation. The p-values were corrected for multiple testing according to the Benjamini-Hochberg method.

Genome browser tracks were created by bedtools genomecov command.

\section{Northern blot analysis of sRNAs}

Northern blot analysis was carried out as described earlier (Baksa et al. 2015) to measure sRNA levels. Three independent biological replicas were analyzed. The signals were quantified using the Image Lab 5.1 software (Bio-Rad) in the following way: the expression values were normalized to the relative U6 or 5S rRNA values (background removed), then the 21 and $27^{\circ} \mathrm{C}$ samples were normalized to the $15^{\circ} \mathrm{C}$ sample value. The primers and probes are listed in Table S5.

\section{Quantitative real-time PCR}

For detection and quantification of miRNAs, a quantitative real-time looped RT-PCR method was used (Varkonyi-Gasic, Wu, Wood, Walton \& Hellens 2007) as described earlier (Baksa et al. 2015).

Quantitative real-time RT-PCR was used to validate predicted differential expression of mRNAs. At first, $1 \mu \mathrm{g}$ total RNA was treated with DNase I enzyme (Thermo Fisher Scientific) following the manufacturer's instructions. High Capacity cDNA Reverse Transcription Kit (Applied Biosystems, Thermo Fisher Scientific) was used for the quantitative conversion of $500 \mathrm{ng}$ DNase treated RNA to single-stranded cDNA. In line with this, another reaction was prepared without enzyme which was used as no reverse transcription control. As reference genes, PP2AA3 (AT1G13320) and ACT7 (AT5G09810)

were used for normalization. The qPCR was performed using FastStart Essential DNA Green Master Mix (Roche) following the manufacturer's instructions. The experiments were carried 
out by using a Roche LightLycler 96 Instrument, and we used the LightCycler 961.1 software for data analysis. Three independent biological replicas were analyzed for each gene. To make the expression values comparable, we normalized the target gene expression to the indicated reference gene, and then we normalized every sample to the $21^{\circ} \mathrm{C}$ sample. The primers and probes are listed in Table S5.

Methylation-sensitive (Chop) PCR

Methylation-sensitive PCRs were carried out according to the protocol provided by Zhang et al. (Zhang et al. 2014) for testing the DNA-methylation state of thermoresponsive siRNA loci. Briefly, the genomic DNA was either digested with the methylation-sensitive restriction enzyme $A l u \mathrm{I}$ or not digested to provide a reference for normalization. After purification, the samples were subjected to quantitative real-time PCR (see above). The signal of the digested template was normalized to the signal of the undigested template from the same sample, and then every sample was normalized to the $15^{\circ} \mathrm{C}$ sample. The used primers are listed in Table S5.

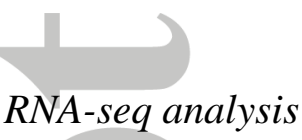

The filtered sequences were mapped to the Arabidopsis thaliana TAIR10 transcriptome (TAIR10_cdna_20101214_updated.fasta) using kallisto 0.44.0, the transcript-level expression table with normalized TPM (transcript per million) values was generated using sleuth 0.28 .0 (Pimentel, Bray, Puente, Melsted \& Pachter 2017). The expression values can be found in Table S4. On separate tabs, we collected the expression of the thermoregulated miRNA target genes (Table S4A), the NF-Y subunit genes (Table S4B), the epigenetics-related genes, and all the TAIR10 transcripts (Table S4D). 


\section{Results and discussion}

High-throughput sequencing of mRNAs, sRNAs and degradome libraries from Arabidopsis thaliana plants grown at different ambient temperatures

We have shown previously that virus-induced gene silencing and transgene silencing is regulated by ambient temperature and being more active at $27{ }^{\circ} \mathrm{C}$ than at $15^{\circ} \mathrm{C}$ (Szittya et al. 2003). This observation suggested that the silencing machinery that regulates endogenous genes is also temperature controlled. To monitor the effect of ambient temperature on endogenous sRNAs and their target genes on a genomic scale, we profiled changes in sRNA and mRNA expression in the same developmental stages of Arabidopsis thaliana Col-0 plants that were grown at three different ambient temperatures (Fig S1; Table S1). Since we showed earlier that plant miRNAs express differently in certain organs/tissues (Pantaleo et al. 2010; Baksa et al. 2015) we monitored the effect of ambient temperature in some parts of the plants (seedling, root, leaf, and flower).

To characterize the different classes of sRNAs, we predicted sRNA-producing loci de novo using ShortStack 3.4 (Johnson et al. 2016) and determined their read length distribution, phasing pattern, strandedness, complexity, overlap with genes or promoters, and whether they derive from hairpins with a potential to be miRNA-producing loci. The raw read counts of these loci were also measured in every sample. In this way, we identified 116,169 sRNA loci. We used DESeq2 (Love et al. 2014) to normalize the expression levels and identify the differentially expressed sRNA loci between temperatures in every tissue separately (Figure 1). We considered those loci differentially expressed which had a mean normalized abundance $>=10$ across all samples in a tissue, changed at least $1.5 \times$ between two temperatures with a q-value $<0.05$ (5\% false discovery rate). Altogether, 704 sRNA loci with temperature dependent expression pattern were identified (Table S2A and B). 
Analysing the overlaps between the sRNA loci and the TAIR10 annotations we noticed an enrichment of tRNA genes (Table S2A). We tested two tRNA genes, At1G06610 (Ala-AGC) and Glu- AT1G09110 (Glu-TTC) for sRNA production (tRNA fragments or tRNA-derived sRNAs) with sRNA northern blots. We were able to confirm that these loci indeed produced abundant sRNAs from the 5' end of the mature tRNA in a temperature dependent fashion (Fig S2A and B). Next, we checked the biogenesis of these tRNA fragments using $d c l$ mutant Arabidopsis plants, and it appears to be DCL2-dependent (Fig S2C). Although, the exact roles of tRNA fragments are yet to be elucidated, accumulating evidence suggests that tRNAderived sRNAs participate in the translational regulation of gene expression under stress conditions. Furthermore, recent studies have demonstrated that tRNAs also serve as a source of sRNAs that possess distinct and varied functions in both plants and animals (Keam \& Hutvagner 2015). Moreover, tRNA fragments were shown to incorporate in AGO complexes, and it is likely that they regulate gene expression post-transcriptionally in a similar fashion as miRNAs do (Loss-Morais, Waterhouse \& Margis 2013).

\section{Identification of temperature-regulated miRNAs and their target genes}

To identify all potential miRNAs in our samples (even previously unidentified ones) we performed a de novo miRNA prediction with ShortStack 3.4 (Johnson et al. 2016) using all sRNA libraries combined. The most abundant sequences (i.e., 5p) and the corresponding antiparallel sequences (i.e., $3 p)$ were collected $(2 \times 90)$ and compared to the 286 nonredundant Arabidopsis thaliana miRNA sequences deposited in miRBase v21 (Kozomara \& Griffiths-Jones 2014). A union of the two sets (378 unique sequences) was created and used for further analysis. Most of the de novo identified miRNA loci matched known miRNA genes, but four putative novel miRNA loci have been identified as well (Fig S3). The expression levels of these loci were very low under our growth conditions. Therefore, these 
potentially novel miRNAs were not included in the subsequent analysis. However, they might be induced under other, more specific conditions like biotic or abiotic stresses or might be expressed in specific cells like meristems or in tissues that we did not sample, for example, seeds.

Next, we identified temperature-regulated miRNAs by filtering for sequences that had maximum abundances more than 40 RPM and changed more than 1.5-fold between different ambient temperatures. This filtering step resulted in 50 temperature-regulated miRNA sequences (Figure 2, Table S2C), including iso-miRs and antiparallel sequences (“star” strands) of known miRNAs. Many of the temperature-regulated miRNA sequences show clear tissue-specific expression pattern, e.g., miR780 expresses mainly in flowers. In many cases, an iso-miR sequence, sometimes the antiparallel sequence ("star" strand) is more abundant than the canonical sequence. One example is the miR169f-3p, which is roughly ten times more abundant in our libraries than the canonical miR169f-5p sequence and is strongly temperature dependent, decreasing at a higher temperature. We also found a few temperatureregulated non-conserved miRNAs. For example, the Arabidopsis-specific miR779 (both the $5 p$ and the $3 p$ sequence with similar, medium-high abundance) was down-regulated at a higher temperature in every tissue, but we could not detect any targets for this miRNA. We used northern blot assay to confirm the results of our bioinformatics analysis in the case of miR156, miR165, miR169, and miR398. Those miRNAs that gave a signal on the northern blot, in most cases were detectable in all of the tissues tested, and they proved to be temperature-regulated (Figure 3, Fig S4). Previously, Lee et al. identified six ambient temperature-responsive miRNAs (miR156, miR163, miR169, miR172, miR398, and miR399) at two different temperatures $\left(16\right.$ and $\left.23^{\circ} \mathrm{C}\right)$ in 8 and 10 days old Arabidopsis seedlings (Lee et al. 2010). The miR156, miR169, miR398, and miR399 were also 
temperature regulated in our samples, despite the fact that we had a different experimental setup compared to Lee's. Some discrepancies can be explained by the differences in the sample collection and detection methodology. While they sampled the plants at the same time (8 and 10-days-old plants), we collected samples at the same developmental stages. For example, our seedling samples were collected at stage 1.04 (Boyes et al. 2001), which was day 9 at $27^{\circ} \mathrm{C}$, day 11 at $21^{\circ} \mathrm{C}$, and day 15 at $15^{\circ} \mathrm{C}$. They collected samples at ZT8 and ZT10, while we collected them at ZT6. They used both microarray and Northern analysis to detect miRNAs, while we used NGS and Northern - e.g., miR172 read number was low, and we filtered it out from the analysis.

In plants, miRNA-mediated mRNA cleavage is highly specific, and miRNAs have been shown to bind with near-perfect complementarity to their mRNA targets, which lead to the slicing of the mRNA between positions 10 and 11 of the AGO1 bound miRNA. As a consequence, the cleaved degradation products are expected to be enriched relative to other, random degradation products along the transcript. We identified the cleavage products of the thermoresponsive and other miRNAs using high-throughput degradome sequencing. We prepared degradome libraries from the same four tissues at the same three temperatures as in the case of small RNA libraries in two biological replicas. The degradome analysis was performed with the CleaveLand4 pipeline (Brousse et al. 2014). To increase the sensitivity of the analysis, we merged all the libraries. The abundance of the sequenced tags was plotted on each transcript, and the cleaved target transcripts have been sorted into five categories $(0,1$, 2, 3 and 4) as it was defined previously in CleaveLand2 (Addo-Quaye, Miller \& Axtell 2009). Namely, Category 0: more than one read at the cleavage position, and this is the most abundant along the transcript; Category 1: the same as Category 0 but there are more than one degradome tags along the transcript with the same abundance as the one at the cleavage site 
(this is quite rare); Category 2: more than one read at the cleavage site above the mean of all the degradome tag abundances, but it is not the highest along the transcript; Category 3: more than one read at the cleavage site, but below or equal to the mean degradome tag abundance along the transcript; Category 4: Just one read at the cleavage position. We retained hits with category 0-2 having a p-value $\leq 0.1$ (Axtell 2018) and an Allen score $<=5$, resulting in 133 miRNA/cleavage target pairs, including 108 category 0 hits (Table S3A and Fig S5). We found 48 thermoregulated miRNA/target pairs (Table S3B) and performed a GO term enrichment analysis with the targets using all genes as a background. We found a significant enrichment for the GO terms of transcription factor activity, CCAAT-binding factor complex, meristem initiation, shoot development and many others (Fig S6, Table S3C). It was not surprising as miRNAs usually target transcription factor genes. When the analysis was performed comparing the thermoregulated miRNA-targets against all the miRNA targets identified, only the category CCAAT-binding complex and its parent categories were found to be enriched (Fig S7, Table S3D). This category consists of the miR169 targeted NF-YA members which suggests that they play an important role in the ambient temperature regulated transcription.

\section{MiR169 is ambient temperature-regulated}

Since the GO enrichment analysis of the thermoregulated miRNA targets put a spotlight on the miR169 and its targets we investigated them in more detail. The miR169 family is evolutionarily conserved in plants, and it has been identified in more than 40 species (Sunkar \& Jagadeeswaran 2008). The miR169 family often makes up the largest miRNA family in plants. In Arabidopsis thaliana, there are four isoforms of miR169 encoded by 14 different genes (Fig S8). The miR169 isoforms show distinct expression patterns during development

(Gonzalez-Ibeas et al. 2011; Sorin et al. 2014), in response to abiotic (Licausi et al. 2011; 
Zhao, Ding, Zhu, Zhang \& Li 2011) or biotic stresses (Singh, Talla \& Qiu 2012), suggesting a functional specialization. The miR169 family target mRNAs encoding subunits A of the Nuclear Factor Y (NF-Y) transcription factor complex. We also identified NF-YA mRNAs that were cleaved by miR169 (Fig S9). These transcription factors (TF) form a conserved heterotrimeric TF complex that is composed of NF-YA, NF-YB and NF-YC subunits (Petroni et al. 2012). The NF-YB and NF-YC subunit contain a histone fold domain, which is very similar to $\mathrm{H} 2 \mathrm{~A}$ and $\mathrm{H} 2 \mathrm{~B}$ core histones. The NF-YA subunit confers sequence-specificity to the complex and recognizes the CCAAT motif in eukaryotic promoters. The Arabidopsis thaliana genome contains $10 \mathrm{NF}-\mathrm{YA}, 10 \mathrm{NF}-\mathrm{YB}$, and $10 \mathrm{NF}-\mathrm{YC}$ genes and they could theoretically combine to form 1000 different complexes (Petroni et al. 2012), not counting the possible alternative transcripts that can code for alternative protein isoforms. Furthermore, the large family of CONSTANS, CONSTANS-LIKE, TOC1-domaincontaining proteins can substitute the NF-YA subunit, making the complex prefer the DNA elements other than CCAAT, for example, CCACA (Gnesutta et al. 2017).

It was shown that in Arabidopsis, an NF-Y complex (composed of NF-YA2, NF-YB2, and NF-YC9) control flowering time by integrating environmental and developmental signals (Hou et al. 2014). In accordance with many previous reports, we found that Arabidopsis plants grown at $15{ }^{\circ} \mathrm{C}$ flowers significantly later than at higher ambient temperatures and in their rosette leaves the expression level of $F T$ is lower than at higher ambient temperatures (Figure 4). The expression level of $F T$ among other factors is also regulated by another NF-Y complex (composed of NF-YA2, NF-YB2, NF-YC3) (Siriwardana et al. 2016). The NF-YA2 transcript is cleaved by miR169h-n-5p, according to our degradome analysis (Fig S9). In line with flowering time and the $F T$ expression level, we found that the NF-YA2 mRNA level anti-correlates with miR169h-n-5p accumulation, being higher at $27{ }^{\circ} \mathrm{C}$ (Figure 4). Some other components of the NF-Y complex also show a temperature-regulated expression pattern 
(Table S4B). However, the B, the C, and some A subunits are apparently independent of miR169 regulation, because we could not detect of their cleavage by this miRNA. Based on these observations we propose that miR169 could mediate ambient temperature signals during inductive photoperiod by regulating $N F-Y A 2$ level to modify flowering time via $F T$ (Figure 9). According to our degradome data, miR169h-n-5p also regulates the expression of the JAZ4 transcriptional regulator by cleaving its mRNA (Fig S9, Table S3B). A subset of JAZ proteins (including JAZ4) interacts with Apetala2 transcription factors Target of Eat1 (TOE1) and TOE2. TOE1 and TOE2 negatively regulate flowering by repressing the transcription of $F T$ (Zhang, Wang, Zeng, Zhang \& Ma 2015). It was also shown that JAZ interaction with TOE1 relieves the repression effect of TOE1 on FT (Zhai et al. 2015). We propose that miR169 could also modulate $F T$ expression level in a temperature-dependent manner by post-transcriptional regulation of $J A Z 4$.

\section{Identification of temperature-regulated 21-nt phasiRNAs}

We identified phasiRNA-producing loci using ShortStack (Figure 5, Table S2D). We found most of the known $T A S$ genes (except $T A S 3 B$ ) and many protein-coding loci that were previously described (e.g. members of the large penta- and tetratricopeptide repeat containing gene family, NBS-LRR genes, cation/H+ exchanger 18) and a few others that have not been described yet (e.g. protochlorophyllide oxidoreductase B, DNAJ heat shock N-terminal domain-containing protein, a plant protein of unknown function, aspartate kinase 3, trypsin family protein with PDZ domain, saccharopine dehydrogenase and bZIP7). It is worth noting that under our conditions, the TAS3 target AUXIN RESPONSE FACTOR 4 (ARF4), and the miR393 target auxin receptor genes (AUXIN SIGNALING F-BOX 2 and 3) are not just cleaved but also produce phasiRNAs, which suggests an amplified silencing of these and maybe other related genes. The expression of many phasiRNA-producing loci is also tissue- 
specific. The majority of the phasiRNA-producing loci were down-regulated with the temperature increasing (Figure 5) suggesting that the level or activity of a common biogenesis component is temperature-sensitive.

\section{Identification of temperature-regulated heterochromatin-associated siRNAs}

We have shown previously that transgene and virus-derived 21-22-nt siRNAs over accumulate at high ambient temperatures (Szittya et al. 2003). This observation suggested that the silencing machinery that is protecting the genome against molecular invaders is more active at $27{ }^{\circ} \mathrm{C}$ than at $15^{\circ} \mathrm{C}$. However, the vast majority of endogenous plant sRNAs are heterochromatin-associated 24-nt siRNAs, therefore we checked the effect of ambient temperature on their accumulation. In our samples, the genomic location and expression level of these loci are stable, and to our surprise are not influenced by ambient temperature. Furthermore, the locations of these 24-nt siRNA loci correlate well with the publicly available methylation data from other experiments (Fig S10). However, there are some temperature-dependent 24-nt siRNA loci at distinct sites which are associated with transposons or intergenic regions (Figure 6 and Table S2E). Such a strongly thermoregulated heterochromatic siRNA-producing locus is located in the promoter region of $Y U C 2$. The YUCCA gene family of Arabidopsis thaliana contains 11 genes and encode flavin monooxygenase-like enzymes that function in tryptophan-dependent auxin biosynthesis (Cheng, Dai \& Zhao 2006). Auxin is a key hormone that regulates many aspects of plant growth and development including floral organ formation and vascular development (Millner 1995). Auxin accumulation coupled with induction of $Y U C$ genes ( $Y U C 8$ and $Y U C 9)$ was shown to be increased in cotyledons upon exposure of Arabidopsis thaliana seedlings to mild high temperature $\left(28-29{ }^{\circ} \mathrm{C}\right)$ (de Wit, Lorrain \& Fankhauser 2014). At high ambient temperature, PIF4 binds to the promoter of YUC8 and stimulates its expression (Franklin et 
al. 2011; Sun, Qi, Li, Chu \& Li 2012). YUC genes are partially redundant, and they are expressed in developmentally distinct spatiotemporal patterns. YUC2 is expressed in young leaf primordia, vasculature and adult leaves (Cheng et al. 2006). At the aforementioned locus in the YUC2 promoter (Locus_77297) the 24-nt siRNAs accumulated to a much lower level at $27{ }^{\circ} \mathrm{C}$ than at $15{ }^{\circ} \mathrm{C}$ or $21^{\circ} \mathrm{C}$ in all tissues. Consistent with the location of the 24 -nt siRNAs, the DNA in the same region is methylated in the leaf (Figure 7), according to publicly available DNA methylation datasets from a previous study (Stroud, Greenberg, Feng, Bernatavichute \& Jacobsen 2013). We tested the temperature-dependency of the CHH methylation at this locus with DNA-methylation-sensitive Chop-qPCR and the expression level of $Y U C 2$ mRNA with RT-qPCR in leaf samples grown at 15, 21 and $27{ }^{\circ} \mathrm{C}$. We found a positive correlation between the level of $\mathrm{CHH}$ methylation of the DNA and the number of 24nt siRNAs, and a negative correlation between DNA methylation and YUC2 expression (Figure 8). This observation suggests that the $Y U C 2$ transcription is under epigenetic control by RNA-directed DNA methylation. The YUC2 promoter does not contain G-box (CACGTG), which is the binding site of PIF4 and other transcription factors (Ezer et al. 2017b) but contains binding sites for the NF-Y complex (CCAAT) and the NF-CO complex (CCACA) around the 24-nt siRNA locus (Figure 7). It suggests that the methylation state and perhaps the chromatin structure at this locus affects the binding of these NF complexes and ultimately the $Y U C 2$ expression, like in the case of $F T$ (Cao et al. 2014). Supporting this hypothesis, a recent study demonstrated that NF-YA2- and NF-YA10-containing complexes could bind the $Y U C 2$ promoter altering the expression of the gene (Zhang, $\mathrm{Hu}, \mathrm{Zhu}, \mathrm{Xu} \&$ Wang 2017).

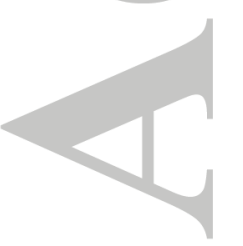


As a summary, we suggest a hypothesis to explain the role of miR169 and its targets, NF-YA and JAZ4 in the regulation of leaf morphology and flowering time at different ambient temperatures (Figure 9). According to this, the level of NF-YA and JAZ4 is higher in the warm due to the downregulation of miR169, resulting in the activation of NF-YA-dependent gene expression, including $F T$ and $Y U C 2$ and the inhibition of TOE action on $F T$ expression by JAZ4. The YUC2 gene expression might have another layer of regulation: the hypomethylation of the DNA at the $Y U C 2$ promoter in the warm may allow more NF-Y complexes and maybe other transcription factors (but not PIF4 or related G-box-binding factors) to bind, resulting in a higher $Y U C 2$ expression and higher auxin level, without a direct PIF4 action. We speculate that the temperature-dependent epigenetic regulation of YUC2 expression by heterochromatic siRNAs and RNA-dependent DNA methylation represents a PIF4-independent regulation of auxin level that may help the plants to adapt to higher ambient temperature by adjusting body shape, flowering time and other auxindependent processes.

\section{Supplementary data}

Table S1. Sequence mapping statistics.

Table S2. Expression of sRNAs.

Table S3. Degradome analysis.

Table S4. Expression of mRNAs.

Table S5. Oligonucleotides used in experiments.

Fig S1. Samples collected for the experiments.

Fig S2. Expression of tRNA-derived sRNA fragments. 
Fig S3. Secondary structures of the newly identified Arabidopsis miRNA precursor RNA molecules.

Fig S4. Northern blot analysis of the thermoregulated miRNAs.

Fig S5. Degradome target plots.

Fig S6. GO term enrichment analysis of the thermoregulated miRNA target genes using all genes as a background.

Fig S7. GO term enrichment analysis of the thermoregulated miRNA target genes using all miRNA target genes as a background.

Fig S8. The mature sequences of the Arabidopsis thaliana miR169 family with the chromosomal location.

Fig S9. Target plots of miR169 targets confirmed by degradome sequencing.

Fig S10. Genomic view of the 24-nt siRNA coverage, methylation density and H2A.Z enrichment within a $280 \mathrm{~kb}$ region on chromosome 1 .

\section{Acknowledgments}

The authors would like to thank Anikó Szigeti for her valuable laboratory assistance during this project. The authors declare that they have no conflict of interest. This work was supported by Hungarian National Research, Development and Innovation Office grant K101793, K-106170 and K-119701.

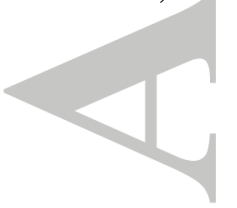




\section{Data availability}

The raw sequencing data have been deposited in the SRA database under the identifier SRP117737. The NCBI BioProject identifier is PRJNA407271. The scripts used for the analysis are available at https://github.com/gyulap/thermoregulated_sRNAs.

\section{References}

Addo-Quaye C., Miller W. \& Axtell M.J. (2009) CleaveLand: a pipeline for using degradome data to find cleaved small RNA targets. Bioinformatics 25, 130-131.

Allen E., Xie Z., Gustafson A.M. \& Carrington J.C. (2005) microRNA-Directed Phasing during Trans-Acting siRNA Biogenesis in Plants. Cell 121, 207-221.

Andrews S. (2010) FastQC: a quality control tool for high throughput sequence data. http://www.bioinformatics.babraham.ac.uk/projects/fastqc/.

Argyris J., Truco M.J., Ochoa O., Knapp S.J., Still D.W., Lenssen G.M., ... Bradford K.J. (2005) Quantitative trait loci associated with seed and seedling traits in Lactuca. TAG. Theoretical and applied genetics. Theoretische und angewandte Genetik 111, 13651376.

Aukerman M.J. \& Sakai H. (2003) Regulation of flowering time and floral organ identity by a MicroRNA and its APETALA2-like target genes. The Plant Cell 15, 2730-2741. Axtell M. (2018) CleaveLand4: Analysis of degradome data to find sliced miRNA and siRNA targets.

Baksa I., Nagy T., Barta E., Havelda Z., Várallyay É., Silhavy D., ... Szittya G. (2015) Identification of Nicotiana benthamiana microRNAs and their targets using high throughput sequencing and degradome analysis. BMC Genomics 16, 1025. 
Baksa I. \& Szittya G. (2017) Identification of ARGONAUTE/Small RNA Cleavage Sites by Degradome Sequencing. Methods in Molecular Biology (Clifton, N.J.) 1640, 113128.

Benjamini Y. \& Hochberg Y. (1995) Controlling the False Discovery Rate: A Practical and Powerful Approach to Multiple Testing. Journal of the Royal Statistical Society. Series B (Methodological) 57, 289-300.

Boyes D.C., Zayed A.M., Ascenzi R., McCaskill A.J., Hoffman N.E., Davis K.R. \& Görlach J. (2001) Growth Stage-Based Phenotypic Analysis of Arabidopsis. The Plant Cell 13, 1499-1510.

Brousse C., Liu Q., Beauclair L., Deremetz A., Axtell M.J. \& Bouché N. (2014) A noncanonical plant microRNA target site. Nucleic Acids Research 42, 5270-5279.

Cao S., Kumimoto R.W., Gnesutta N., Calogero A.M., Mantovani R. \& Holt B.F. (2014) A Distal CCAAT/NUCLEAR FACTOR Y Complex Promotes Chromatin Looping at the FLOWERING LOCUS T Promoter and Regulates the Timing of Flowering in Arabidopsis. The Plant Cell Online 26, 1009-1017.

Carthew R.W. \& Sontheimer E.J. (2009) Origins and Mechanisms of miRNAs and siRNAs. Cell 136, 642-655.

Chen X. (2004) A microRNA as a translational repressor of APETALA2 in Arabidopsis flower development. Science 303, 2022-2025.

Cheng Y., Dai X. \& Zhao Y. (2006) Auxin biosynthesis by the YUCCA flavin monooxygenases controls the formation of floral organs and vascular tissues in Arabidopsis. Genes \& Development 20, 1790-1799.

Ding S.-W. \& Voinnet O. (2007) Antiviral immunity directed by small RNAs. Cell 130, 413426. 
Du Z., Zhou X., Ling Y., Zhang Z. \& Su Z. (2010) agriGO: a GO analysis toolkit for the agricultural community. Nucleic Acids Research 38, W64-W70.

Ezer D., Jung J.-H., Lan H., Biswas S., Gregoire L., Box M.S., .. Wigge P.A. (2017a) The Evening Complex coordinates environmental and endogenous signals in Arabidopsis. Nature plants $\mathbf{3}, 17087$.

Ezer D., Shepherd S.J.K., Brestovitsky A., Dickinson P., Cortijo S., Charoensawan V., ... Wigge P.A. (2017b) The G-Box Transcriptional Regulatory Code in Arabidopsis1. Plant Physiology 175, 628-640.

Fei Q., Xia R. \& Meyers B.C. (2013) Phased, secondary, small interfering RNAs in posttranscriptional regulatory networks. The Plant Cell 25, 2400-2415.

Franklin K.A., Lee S.H., Patel D., Kumar S.V., Spartz A.K., Gu C., .. Gray W.M. (2011) PHYTOCHROME-INTERACTING FACTOR 4 (PIF4) regulates auxin biosynthesis at high temperature. Proceedings of the National Academy of Sciences 108, 2023120235.

Gasciolli V., Mallory A.C., Bartel D.P. \& Vaucheret H. (2005) Partially redundant functions of Arabidopsis DICER-like enzymes and a role for DCL4 in producing trans-acting siRNAs. Current Biology 15, 1494-1500.

Gnesutta N., Kumimoto R.W., Swain S., Chiara M., Siriwardana C., Horner D.S., ...

Mantovani R. (2017) CONSTANS Imparts DNA Sequence Specificity to the Histone Fold NF-YB/NF-YC Dimer. The Plant Cell 29, 1516-1532.

Gonzalez-Ibeas D., Blanca J., Donaire L., Saladié M., Mascarell-Creus A., Cano-Delgado A., Aranda M.A. (2011) Analysis of the melon (Cucumis melo) small RNAome by high-throughput pyrosequencing. BMC genomics 12, 393.

Guo Q., Qu X. \& Jin W. (2015) PhaseTank: genome-wide computational identification of phasiRNAs and their regulatory cascades. Bioinformatics 31, 284-286. 
Hou X., Zhou J., Liu C., Liu L., Shen L. \& Yu H. (2014) Nuclear factor Y-mediated H3K27me3 demethylation of the SOC1 locus orchestrates flowering responses of Arabidopsis. Nature Communications 5, 4601.

Johnson N.R., Yeoh J.M., Coruh C. \& Axtell M.J. (2016) Improved Placement of Multimapping Small RNAs. G3: Genes|Genomes|Genetics 6, 2103-2111.

Jung J.-H., Domijan M., Klose C., Biswas S., Ezer D., Gao M., ... Wigge P.A. (2016)

Phytochromes function as thermosensors in Arabidopsis. Science 354, 886-889.

Kasschau K.D., Fahlgren N., Chapman E.J., Sullivan C.M., Cumbie J.S., Givan S.A. \& Carrington J.C. (2007) Genome-wide profiling and analysis of Arabidopsis siRNAs. PLoS biology 5, e57.

Keam S.P. \& Hutvagner G. (2015) tRNA-Derived Fragments (tRFs): Emerging New Roles for an Ancient RNA in the Regulation of Gene Expression. Life 5, 1638-1651.

Kozomara A. \& Griffiths-Jones S. (2014) miRBase: annotating high confidence microRNAs using deep sequencing data. Nucleic Acids Research 42, D68-73.

Kumar S.V. \& Wigge P.A. (2010) H2A.Z-containing nucleosomes mediate the thermosensory response in Arabidopsis. Cell 140, 136-147.

Lam F.H., Steger D.J. \& O’Shea E.K. (2008) Chromatin decouples promoter threshold from dynamic range. Nature 453, 246-250.

Lee H., Yoo S.J., Lee J.H., Kim W., Yoo S.K., Fitzgerald H., ... Ahn J.H. (2010) Genetic framework for flowering-time regulation by ambient temperature-responsive miRNAs in Arabidopsis. Nucleic Acids Research 38, 3081-3093.

Legris M., Klose C., Burgie E.S., Rojas C.C.R., Neme M., Hiltbrunner A., ... Casal J.J. (2016) Phytochrome B integrates light and temperature signals in Arabidopsis.

Science 354, 897-900. 
Li H., Handsaker B., Wysoker A., Fennell T., Ruan J., Homer N., ... 1000 Genome Project Data Processing Subgroup (2009) The Sequence Alignment/Map format and SAMtools. Bioinformatics 25, 2078-2079.

Licausi F., Weits D.A., Pant B.D., Scheible W.-R., Geigenberger P. \& van Dongen J.T. (2011) Hypoxia responsive gene expression is mediated by various subsets of transcription factors and miRNAs that are determined by the actual oxygen availability. The New Phytologist 190, 442-456.

Llave C., Xie Z., Kasschau K.D. \& Carrington J.C. (2002) Cleavage of Scarecrow-like mRNA targets directed by a class of Arabidopsis miRNA. Science 297, 2053-2056.

Loss-Morais G., Waterhouse P.M. \& Margis R. (2013) Description of plant tRNA-derived RNA fragments (tRFs) associated with argonaute and identification of their putative targets. Biology Direct 8, 6.

Love M.I., Huber W. \& Anders S. (2014) Moderated estimation of fold change and dispersion for RNA-seq data with DESeq2. Genome Biology 15, 550.

Martin M. (2011) Cutadapt removes adapter sequences from high-throughput sequencing reads. EMBnet.journal 17, 10-12.

Matzke M.A., Kanno T. \& Matzke A.J.M. (2015) RNA-Directed DNA Methylation: The Evolution of a Complex Epigenetic Pathway in Flowering Plants. Annual Review of Plant Biology 66, 243-267.

McCormick K.P., Willmann M.R. \& Meyers B.C. (2011) Experimental design, preprocessing, normalization and differential expression analysis of small RNA sequencing experiments. Silence 2, 2.

Millner P.A. (1995) The auxin signal. Current Opinion in Cell Biology 7, 224-231.

Mosher R.A., Schwach F., Studholme D. \& Baulcombe D.C. (2008) PolIVb influences RNAdirected DNA methylation independently of its role in siRNA biogenesis. 
Proceedings of the National Academy of Sciences of the United States of America $\mathbf{1 0 5}, 3145-3150$.

Pantaleo V., Szittya G., Moxon S., Miozzi L., Moulton V., Dalmay T. \& Burgyan J. (2010) Identification of grapevine microRNAs and their targets using high-throughput sequencing and degradome analysis. The Plant Journal 62, 960-976.

Peragine A., Yoshikawa M., Wu G., Albrecht H.L. \& Poethig R.S. (2004) SGS3 and SGS2/SDE1/RDR6 are required for juvenile development and the production of transacting siRNAs in Arabidopsis. Genes \& Development 18, 2368-2379.

Petroni K., Kumimoto R.W., Gnesutta N., Calvenzani V., Fornari M., Tonelli C., ... Mantovani R. (2012) The Promiscuous Life of Plant NUCLEAR FACTOR Y Transcription Factors. The Plant Cell 24, 4777-4792.

Pimentel H., Bray N.L., Puente S., Melsted P. \& Pachter L. (2017) Differential analysis of RNA-seq incorporating quantification uncertainty. Nature Methods 14, 687-690.

Quinlan A.R. \& Hall I.M. (2010) BEDTools: a flexible suite of utilities for comparing genomic features. Bioinformatics 26, 841-842.

Quint M., Delker C., Franklin K.A., Wigge P.A., Halliday K.J. \& Zanten M. van (2016) Molecular and genetic control of plant thermomorphogenesis. Nature Plants 2 , nplants2015190.

Rogers K. \& Chen X. (2013) Biogenesis, turnover, and mode of action of plant microRNAs. The Plant Cell 25, 2383-2399.

Segal E. \& Widom J. (2009) From DNA sequence to transcriptional behaviour: a quantitative approach. Nature Reviews. Genetics 10, 443-456.

Singh K., Talla A. \& Qiu W. (2012) Small RNA profiling of virus-infected grapevines: evidences for virus infection-associated and variety-specific miRNAs. Functional \& Integrative Genomics 12, 659-669. 
Siriwardana C.L., Gnesutta N., Kumimoto R.W., Jones D.S., Myers Z.A., Mantovani R. \& Holt B.F. (2016) NUCLEAR FACTOR Y, Subunit A (NF-YA) Proteins Positively Regulate Flowering and Act Through FLOWERING LOCUS T. PLoS genetics 12, e1006496.

Sorin C., Declerck M., Christ A., Blein T., Ma L., Lelandais-Brière C., ... Hartmann C. (2014) A miR169 isoform regulates specific NF-YA targets and root architecture in Arabidopsis. The New Phytologist 202, 1197-1211.

Stocks M.B., Moxon S., Mapleson D., Woolfenden H.C., Mohorianu I., Folkes L., ... Moulton V. (2012) The UEA sRNA workbench: a suite of tools for analysing and visualizing next generation sequencing microRNA and small RNA datasets.

Bioinformatics 28, 2059-2061.

Stroud H., Greenberg M.V.C., Feng S., Bernatavichute Y.V. \& Jacobsen S.E. (2013)

Comprehensive analysis of silencing mutants reveals complex regulation of the Arabidopsis methylome. Cell 152, 352-364.

Sun J., Qi L., Li Y., Chu J. \& Li C. (2012) PIF4-mediated activation of YUCCA8 expression integrates temperature into the auxin pathway in regulating arabidopsis hypocotyl growth. PLoS genetics 8, e1002594.

Sunkar R. \& Jagadeeswaran G. (2008) In silico identification of conserved microRNAs in large number of diverse plant species. BMC Plant Biology 8, 37.

Szittya G., Silhavy D., Molnár A., Havelda Z., Lovas Á., Lakatos L., .. Burgyán J. (2003) Low temperature inhibits RNA silencing-mediated defence by the control of siRNA generation. The EMBO Journal 22, 633-640.

Varkonyi-Gasic E., Wu R., Wood M., Walton E.F. \& Hellens R.P. (2007) Protocol: a highly sensitive RT-PCR method for detection and quantification of microRNAs. Plant Methods 3, 12. 
Vazquez F., Vaucheret H., Rajagopalan R., Lepers C., Gasciolli V., Mallory A.C., ... Crété P. (2004) Endogenous trans-acting siRNAs regulate the accumulation of Arabidopsis mRNAs. Molecular Cell 16, 69-79.

Voinnet O. (2009) Origin, biogenesis, and activity of plant microRNAs. Cell 136, 669-687.

White J.L. \& Kaper J.M. (1989) A simple method for detection of viral satellite RNAs in small plant tissue samples. Journal of Virological Methods 23, 83-93.

Wierzbicki A.T., Ream T.S., Haag J.R. \& Pikaard C.S. (2009) RNA polymerase V transcription guides ARGONAUTE4 to chromatin. Nature Genetics 41, 630-634.

de Wit M., Lorrain S. \& Fankhauser C. (2014) Auxin-mediated plant architectural changes in response to shade and high temperature. Physiologia Plantarum 151, 13-24.

Xie Z., Allen E., Wilken A. \& Carrington J.C. (2005) DICER-LIKE 4 functions in transacting small interfering RNA biogenesis and vegetative phase change in Arabidopsis thaliana. Proceedings of the National Academy of Sciences 102, 12984-12989.

Yoshikawa M., Peragine A., Park M.Y. \& Poethig R.S. (2005) A pathway for the biogenesis of trans-acting siRNAs in Arabidopsis. Genes \& Development 19, 2164-2175.

Zhai J., Jeong D.-H., Paoli E.D., Park S., Rosen B.D., Li Y., ... Meyers B.C. (2011) MicroRNAs as master regulators of the plant NB-LRR defense gene family via the production of phased, trans-acting siRNAs. Genes \& Development 25, 2540-2553.

Zhai Q., Zhang X., Wu F., Feng H., Deng L., Xu L., ... Li C. (2015) Transcriptional Mechanism of Jasmonate Receptor COI1-Mediated Delay of Flowering Time in Arabidopsis. The Plant Cell 27, 2814-2828.

Zhang B., Wang L., Zeng L., Zhang C. \& Ma H. (2015) Arabidopsis TOE proteins convey a photoperiodic signal to antagonize CONSTANS and regulate flowering time. Genes \& Development 29, 975-987. 
Zhang H., Tang K., Wang B., Duan C.-G., Lang Z. \& Zhu J.-K. (2014) Protocol: a beginner's guide to the analysis of RNA-directed DNA methylation in plants. Plant Methods $\mathbf{1 0}$, 18.

Zhang M., Hu X., Zhu M., Xu M. \& Wang L. (2017) Transcription factors NF-YA2 and NFYA10 regulate leaf growth via auxin signaling in Arabidopsis. Scientific Reports 7.

Zhang X., Henderson I.R., Lu C., Green P.J. \& Jacobsen S.E. (2007) Role of RNA polymerase IV in plant small RNA metabolism. Proceedings of the National Academy of Sciences 104, 4536-4541.

Zhao M., Ding H., Zhu J.-K., Zhang F. \& Li W.-X. (2011) Involvement of miR169 in the nitrogen-starvation responses in Arabidopsis. The New phytologist 190, 906-915.

Zhong X., Du J., Hale C.J., Gallego-Bartolome J., Feng S., Vashisht A.A., ... Jacobsen S.E. (2014) Molecular mechanism of action of plant DRM de novo DNA methyltransferases. Cell 157, 1050-1060.

Zhou M. \& Law J.A. (2015) RNA Pol IV and V in gene silencing: Rebel polymerases evolving away from Pol II's rules. Current Opinion in Plant Biology 27, 154-164.

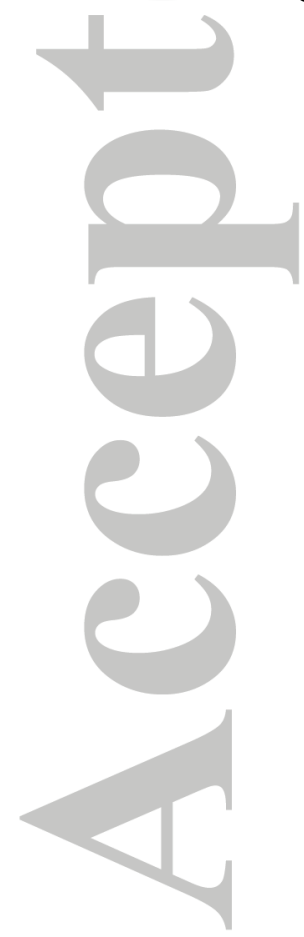



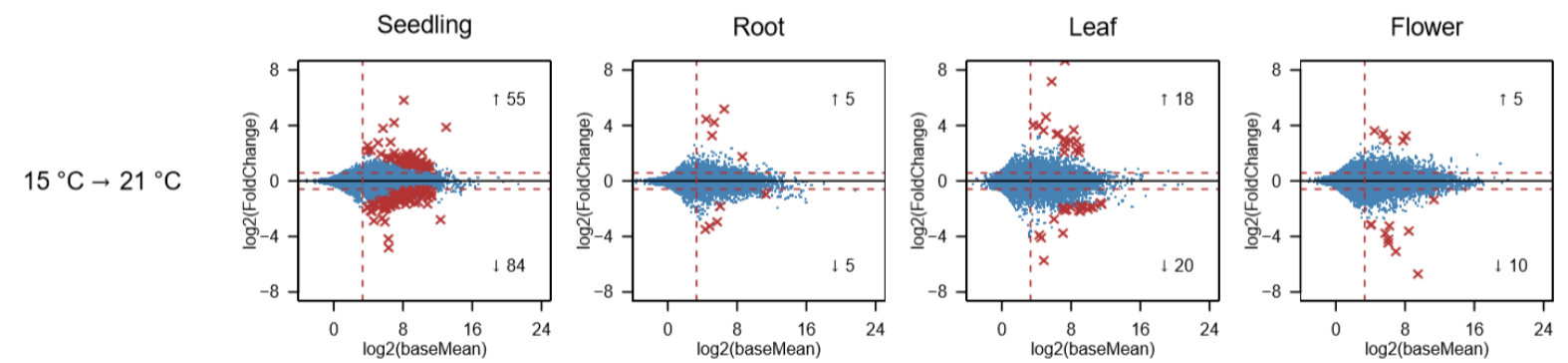

$21^{\circ} \mathrm{C} \rightarrow 27^{\circ} \mathrm{C}$
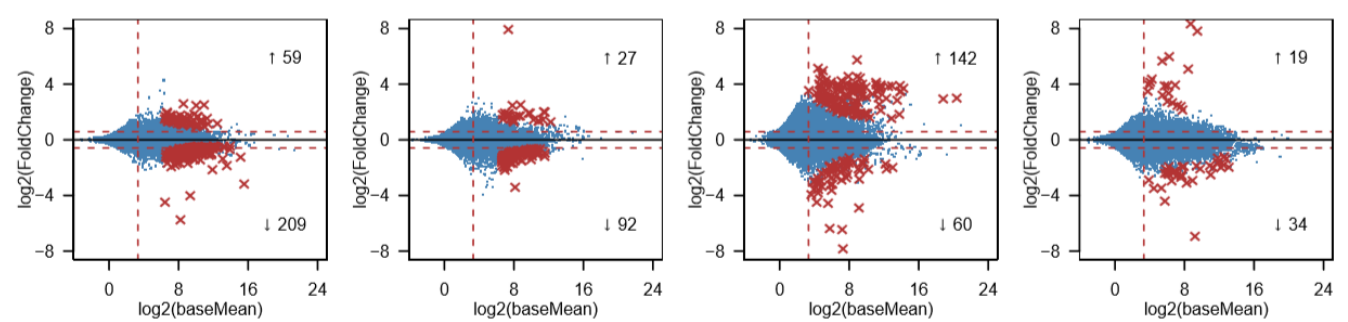

Figure 1. Differential expression of small RNA loci between ambient temperatures in different tissues.

MA-plots show the distribution of expression fold-changes (in $\log 2$ scale) as a function of average expression (the baseMean value of DESeq2 results) of every locus in the compared samples. Positive $\log 2 \mathrm{FC}$ values mean that the expression of the given locus was higher at the higher temperature, while the negative $\log 2 \mathrm{FC}$ value means that it was lower at the higher temperature. Locus expressions were considered changed (red crosses) when the absolute value of expression fold-change between the two investigated conditions was at least $1.5 \times$, the normalized abundance was at least 10 , and the adjusted p-value was lower than 0.05 (5\% false discovery rate). The remaining loci were depicted as blue dots. We identified 116,169 small RNA loci, of which $704(0.6 \%)$ changed altogether in the four tissues and at the three temperatures. More information about these loci can be found in Table S2. 


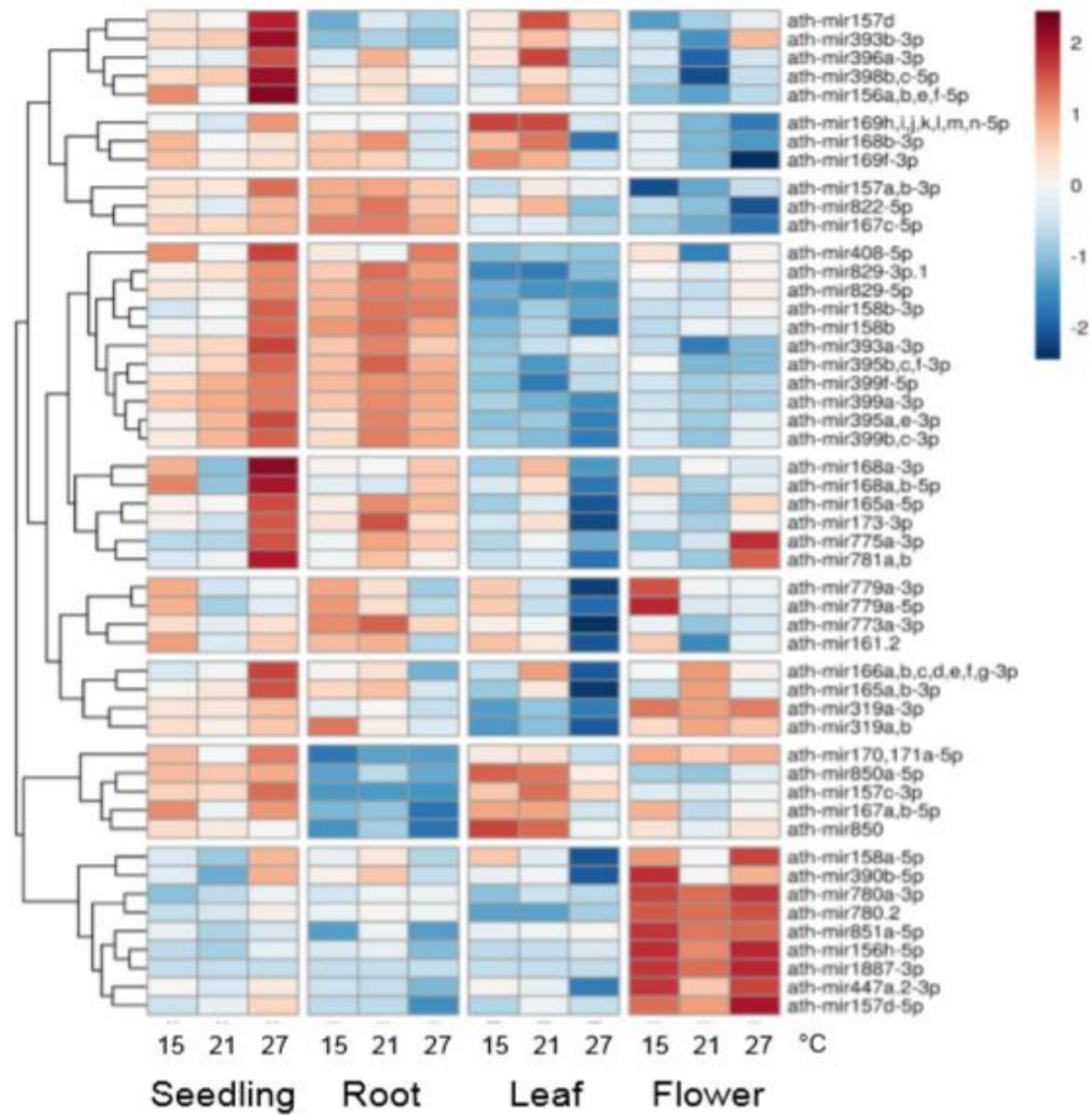

Figure 2. Expression of thermoregulated miRNAs in different tissues.

Nonredundant miRNA sequences from miRBase v21 and the mature and star sequences of

ShortStack-predicted miRNA loci were merged, and their differential expression upon

temperature change was measured. Only sequences with more than 40 RPM maximum value

in any samples that changed at least $1.5 \times$ between temperatures were kept and listed. The colors represent z-scores, which shows how many standard deviations the given value is above or below from the mean of all the values in the row. 


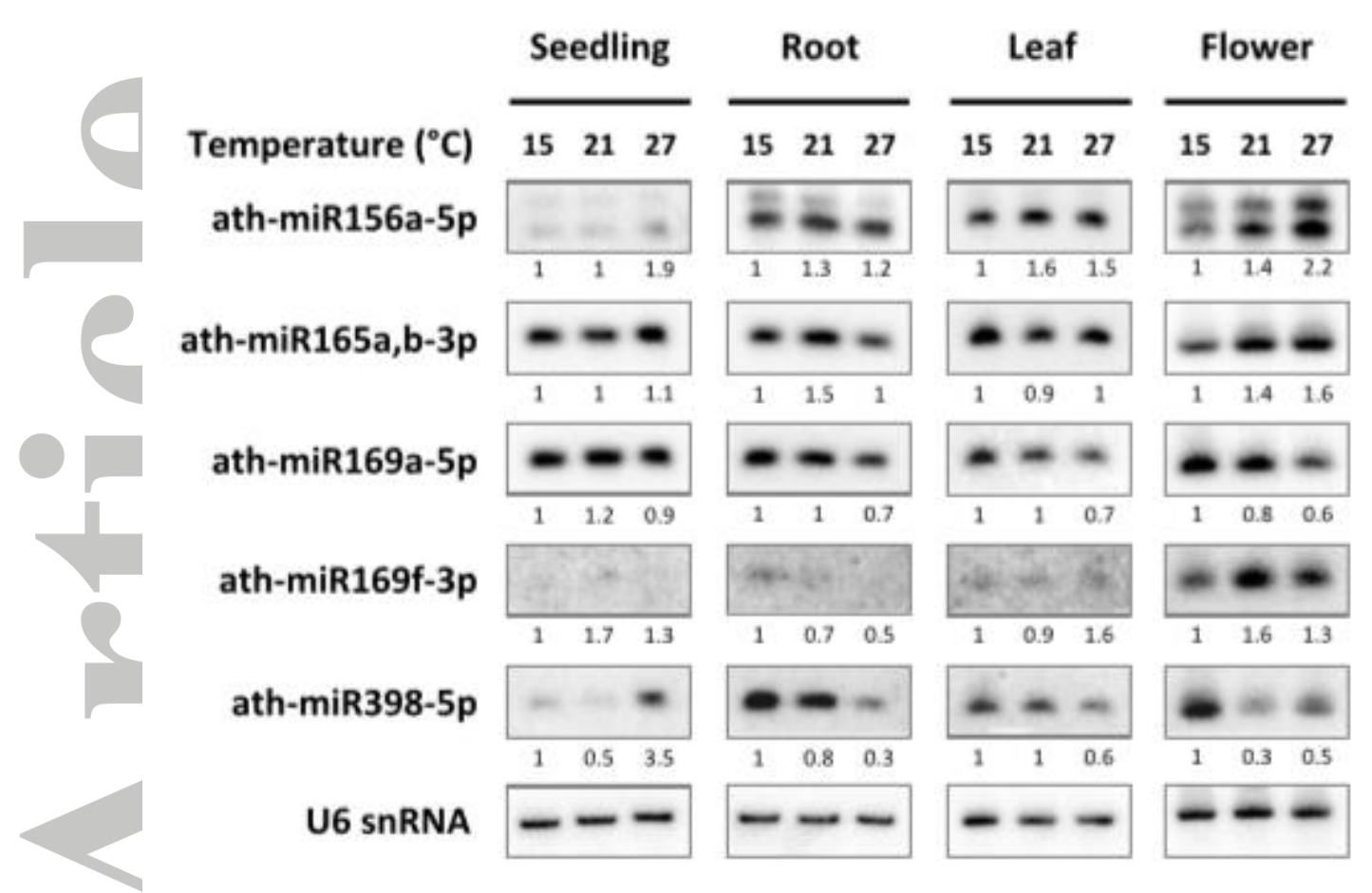

Figure 3. Northern blot analysis of selected temperature-regulated miRNAs in different tissues.

Total RNA was extracted from different tissues of Arabidopsis thaliana plants as described in the Materials and Methods section. The RNA was separated on PAGE and transferred to nylon membranes for Northern blot analysis. Oligonucleotide probes were used to detect specific miRNAs, and a $U 6$-specific probe was used to detect $U 6$ RNA as a loading control. The expression values were measured by densitometry. First, the values were normalized to the corresponding loading control value, then to the $15^{\circ} \mathrm{C}$ sample value. The analysis was performed on three biological replicas, here we show the results of one representative replica. The results of the other two replicas are shown in Fig S4A. 


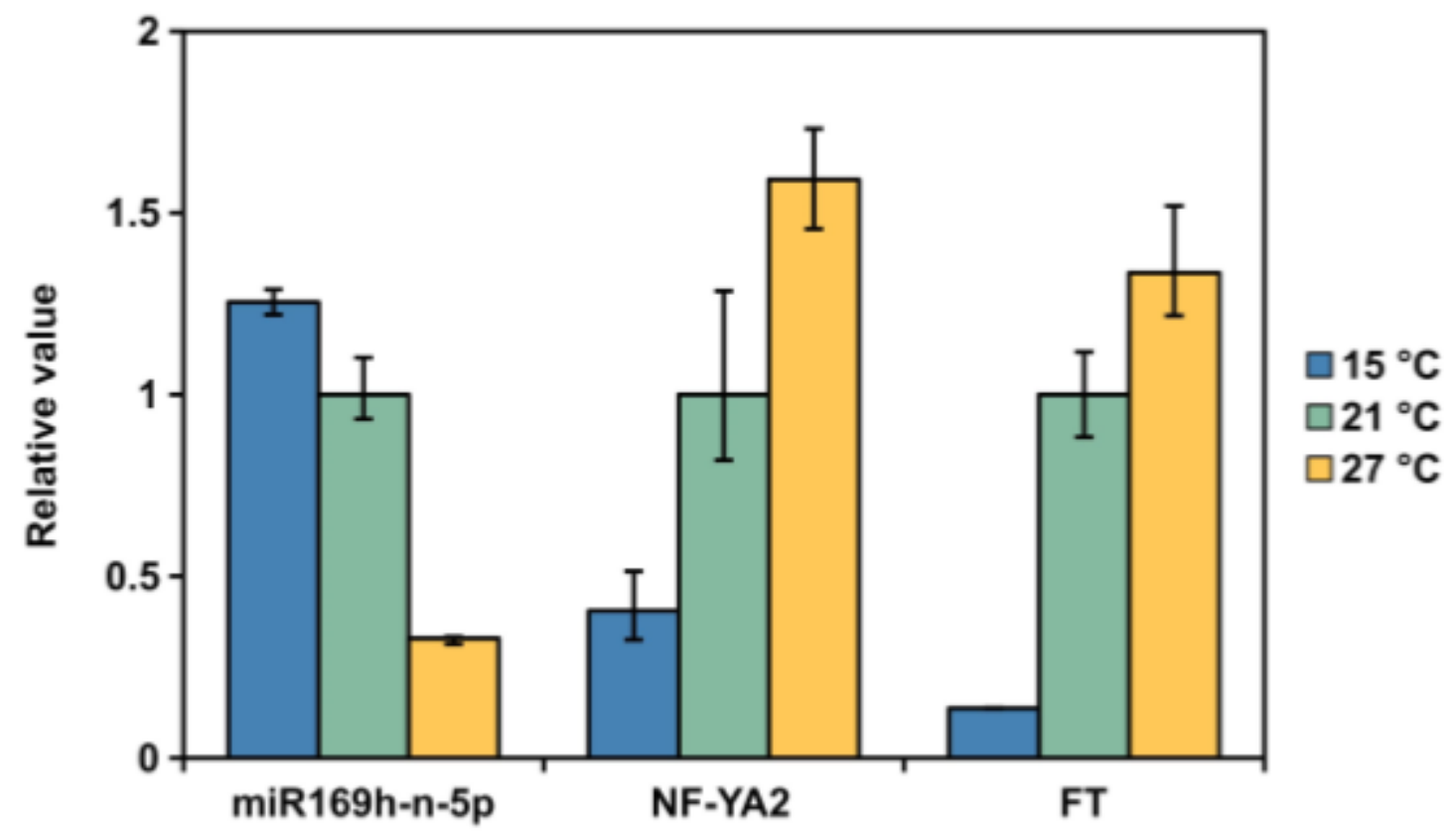

Figure 4. Ambient temperature regulation of miR169h-n-5p, NF-YA2, and FT.

The miR169h-n-5p level is higher at high ambient temperature. $N F-Y A 2$ is down-regulated by miR169h-n-5p at low ambient temperature. The transcription of FT is dependent on NF-Y heterotrimeric protein complexes. The increased $N F-Y A 2$ level at high ambient temperature contributes to the increase of $F T$ level, which results in early flowering. To validate the sequencing results, we prepared RNA samples from three biological replicas at ZT6 (zeitgeber time, 6 hours after lights on) from the leaves of Arabidopsis thaliana plants grown in pots in a controlled growth chamber under long day condition (16L:8D). The miR169h-n$5 \mathrm{p}$ expression values were normalized to the $U 6$ expression, the $N F-Y A 2$ expression values were normalized to the $A C T 7$ expression, while the $F T$ expression values were normalized to the $P P 2 A$ expression. After this, every sample was normalized to the $21^{\circ} \mathrm{C}$ sample. The error bars represent the minimum and the maximum values. 


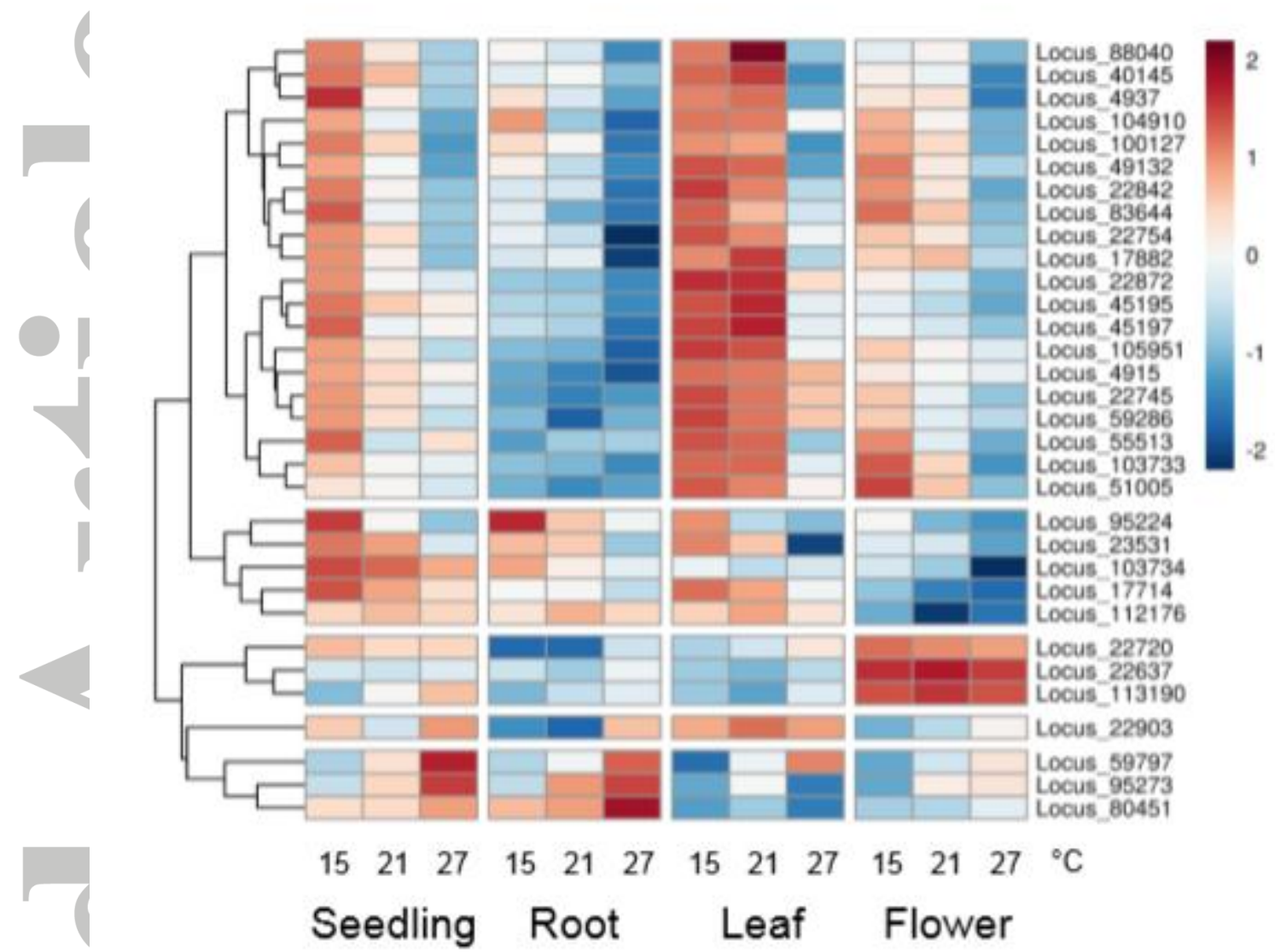

Figure 5. Expression of the 21-nt phasiRNA-producing loci in different tissues.

The 21-nt phasiRNA-producing loci were predicted with ShortStack. Only loci with

PhaseScore $\geq 25$ were retained, and their expression was illustrated on a heat map. Additional information about these loci can be found in Table S2D. Most of the phasiRNA-producing loci are downregulated with the temperature increasing, especially in the leaf. The colors represent z-scores, which shows how many standard deviations the given value is above or below from the mean of all the values in the row. 

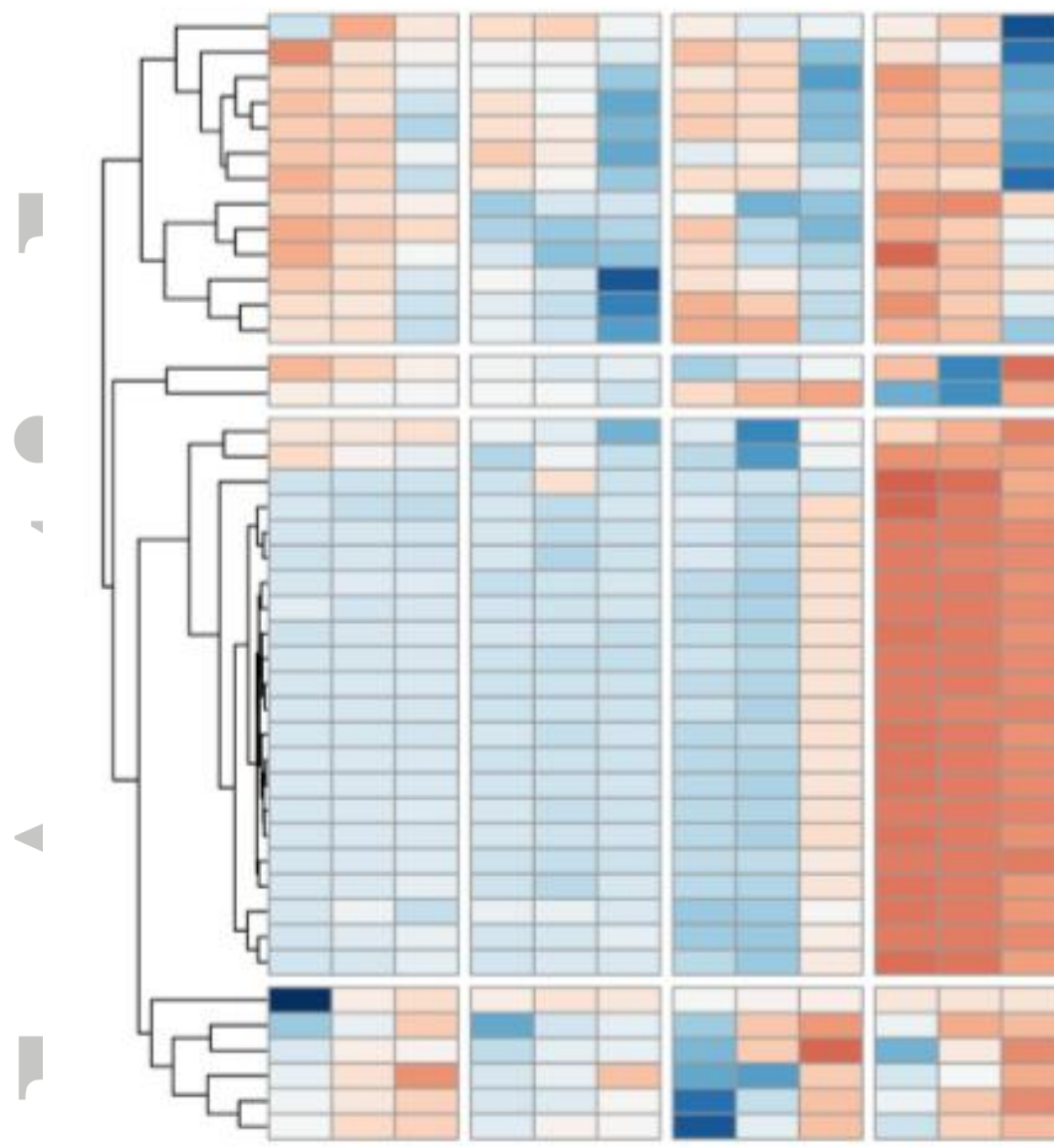

Locus 73298

Locus 19277

Locus 63907

Locus 22532

Locus 77297

Locus 84401

Locus 42244

Locus 101120

Locus 74299

cocus 74522

ocus 273

Locus 18128

Locus 103419

Locus_39319

Locus 74426

Locus 82252

Locus 94508

Locus 24248

Locus_12174

Locus 40407

Locus 59607

Locus 59607

Locus 9774

Locus 97748

Locus 58351

Locus 5097

Locus 83161

Locus 83841

Locus 84476

Locus 22905

Locus 24043

Locus 111500

Locus 11053

Locus 88654

Locus_67622

Locus_82691

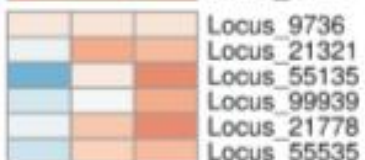

$\begin{array}{lllllllllllll}15 & 21 & 27 & 15 & 21 & 27 & 15 & 21 & 27 & 15 & 21 & 27 & { }^{\circ} \mathrm{C}\end{array}$

\section{Seedling Root Leaf Flower}

Figure 6. Expression of the thermoregulated 24-nt siRNA-producing loci in different

\section{tissues.}

ShortStack-predicted loci with a dominant size class of 24-nt with an average expression level of at least 100, that changed at least $5 \times$ between temperatures are shown. Locus_77297 was identified in the $Y U C 2$ promoter which was consistently downregulated at high ambient temperature in all the four tissues (see Figure 7). The colors represent z-scores, which shows how many standard deviations the given value is above or below from the mean of all the values in the row. 


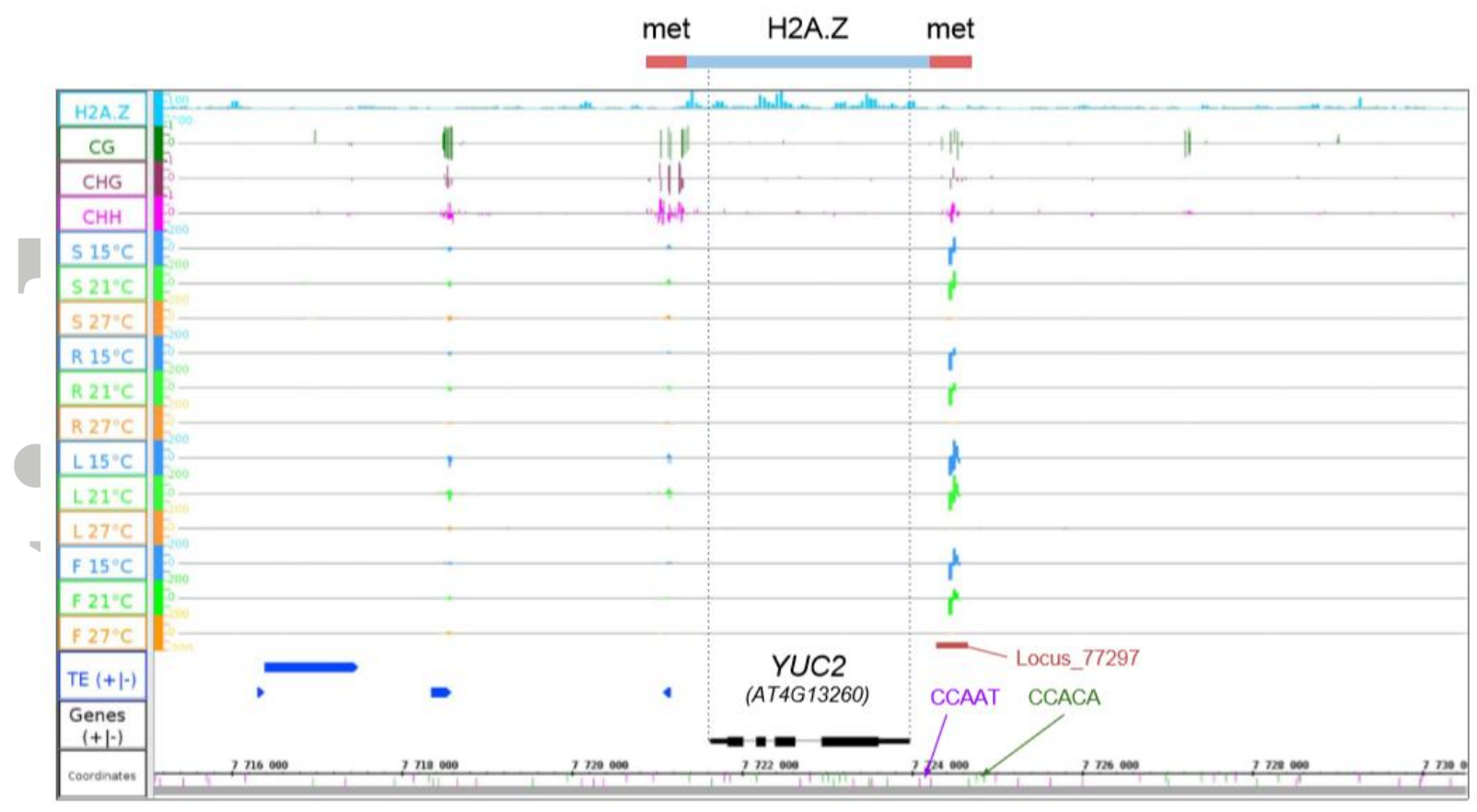

Figure 7. Genomic view of the 24-nt siRNA coverage, methylation density and H2A.Z enrichment in the vicinity of the YUC2 gene.

The normalized coverage data for the 24-nt siRNAs in the seedling (S), root (R), leaf (L), and flower $(\mathrm{F})$ at 15,21 and $27^{\circ} \mathrm{C}$ were visualized in the Integrated Genome Browser.

Methylation data were taken from GSM980986; the H2A.Z enrichment data derive from GSM954590. The thermoregulated Locus_77297 was identified in the YUC2 promoter spanning 263-681 bp upstream of the transcription start site. NF-YA2 and CO-containing NF heterotrimer binding sites along the chromosome are represented by purple and green tick marks, respectively. 

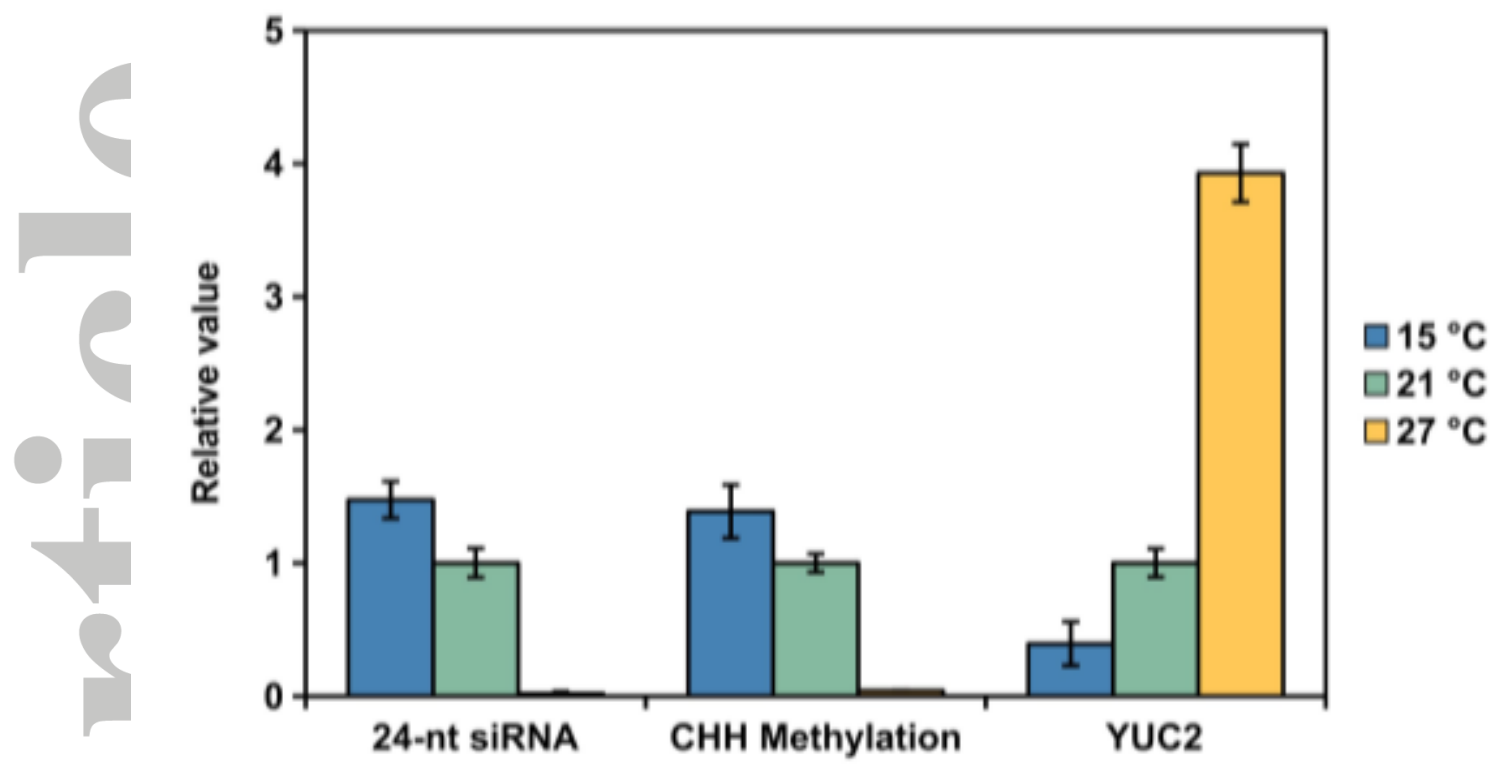

Figure 8. The $Y U C 2$ auxin biosynthesis gene expression is under a possible temperaturedependent epigenetic regulation.

We observed much lower 24-nt siRNA levels at $27{ }^{\circ} \mathrm{C}$ than at 15 or $21{ }^{\circ} \mathrm{C}$ in all the four tissues (see Figure 7), but here we show only the leaf data. The expression pattern of the 24nt siRNAs in the leaf positively correlated with the $\mathrm{CHH}$ methylation state at the same site and negatively correlated with $Y U C 2$ mRNA expression. First, the siRNA read counts were normalized by DESeq2, the Chop-qPCR values of the AluI-digested samples were normalized to the undigested control, while the $Y U C 2$ qPCR values were normalized to $P P 2 A$. Second, all values were scaled to the mean value of the $21^{\circ} \mathrm{C}$ sample. All measurements represent two biological replicas. The error bars denote the minimum and maximum values. 

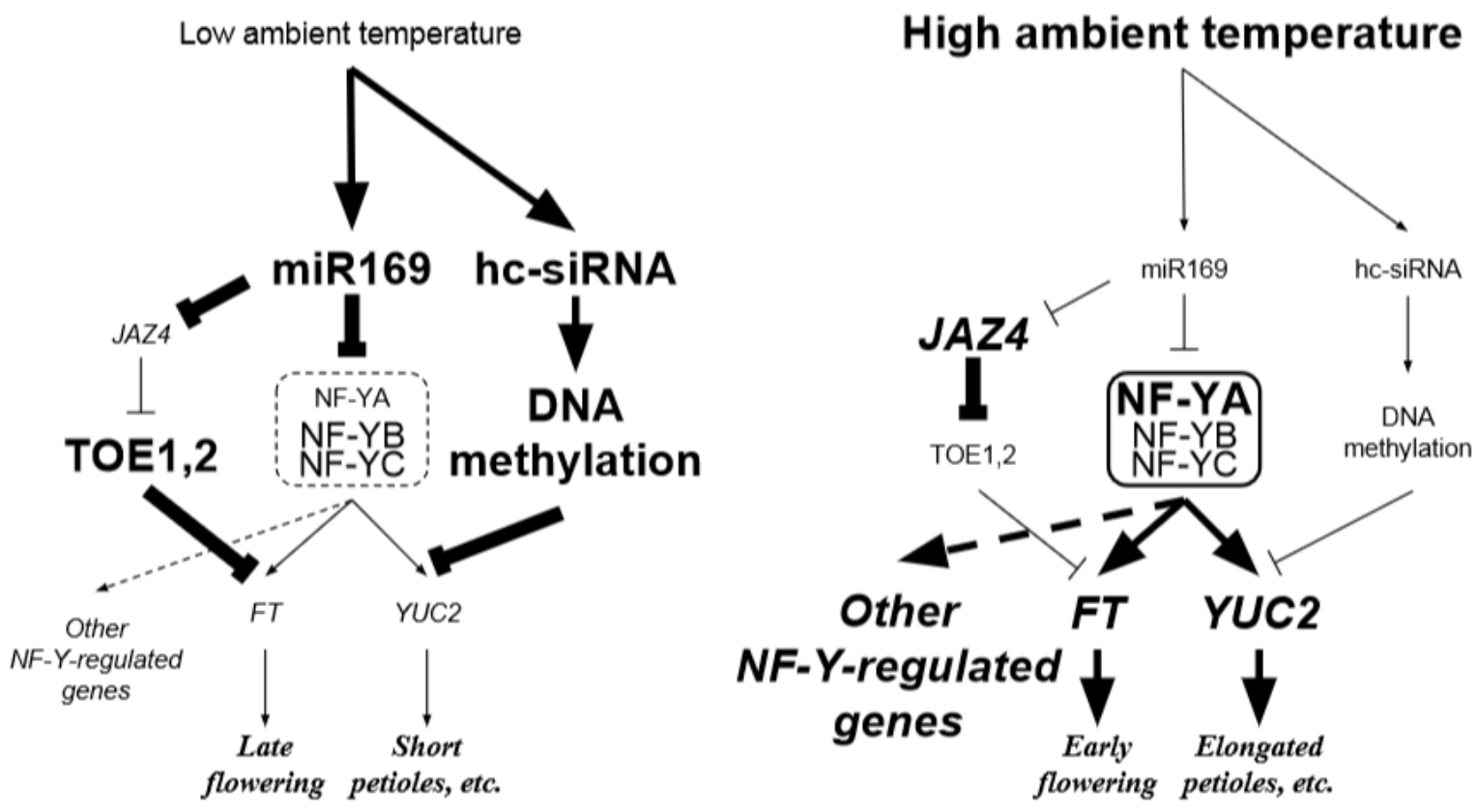

Figure 9. A proposed model for the role of small RNAs in the regulation of flowering time and leaf morphology.

MiR169 can affect the expression of various genes that are dependent on the NF-YAcontaining transcriptional regulators. It was previously shown that certain NF-Y complexes bind to the promoter of the flowering time regulator gene $F T$ (Cao et al. 2014) and the auxin biosynthesis gene $Y U C 2$ (Zhang et al. 2017) resulting in altered flowering time and leaf morphogenesis. A 24-nt siRNA-producing locus in the promoter of the $Y U C 2$ may block the binding of NF-Y and potentially other transcription factors in a temperature-dependent manner. At low temperature, the level of miR169 and the 24-nt hc-siRNA is high, which results in a low concentration of active NF-Y complex and JAZ4, a high concentration of TOE1 and TOE2, and a methylated YUC2 promoter. In the absence of NF-Y the expression of NF-Y-dependent genes, including FT and YUC2 remains low. Furthermore, the $F T$ expression is inhibited by TOEs causing delayed flowering (Zhang et al. 2015; Zhai et al. 
2015). On the other hand, at high ambient temperature, the miR169 level drops, so as the hcsiRNA and the methylation level at the $Y U C 2$ promoter. In parallel, the NF-YA and the NFY-dependent gene expression rises. JAZ4 inhibits the repressor activity of TOEs so that the FT level can rise (Zhai et al. 2015). The high level of FT induces flowering, while the elevated level of $Y U C 2$ modulates leaf morphology by increasing the auxin concentration and promoting cell elongation.

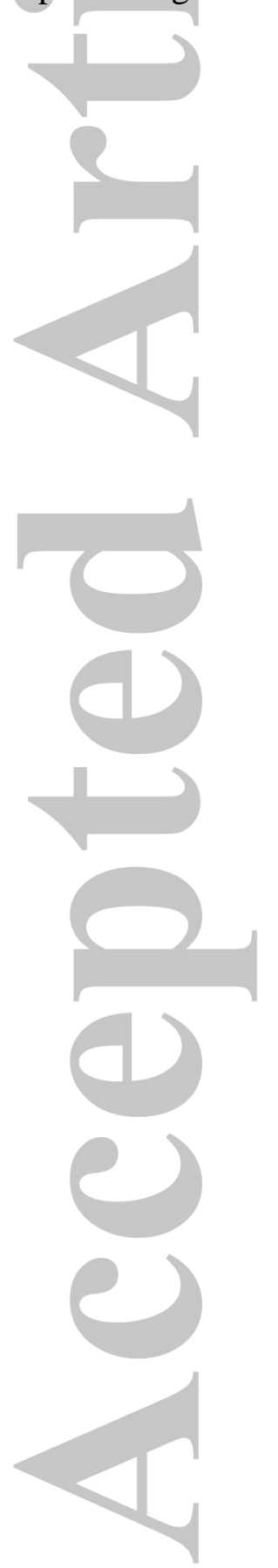

